\title{
Esclerodermia localizada na criança: aspectos clínicos, diagnósticos e terapêuticos
}

\section{Localized scleroderma in children: clinical, diagnostic and therapeutic aspects}

\author{
Pedro C. Q. Zancanaro ${ }^{1}$ \\ Luisimara T. Garcia ${ }^{3}$
}

\author{
Adriana R. Isaac ${ }^{2}$ \\ Izelda M. C. Costa
}

\begin{abstract}
Resumo: A esclerodermia localizada, ou morféia, acomete crianças em idade escolar e, em geral, é autolimitada, apesar de localmente desfiguradora. A literatura descreve inúmeros fatores etiopatogênicos, bem como modalidades de investigação e tratamento. Este artigo reúne os estudos mais recentes e discute sua aplicação clínica.

Palavras-chave: Criança; Esclerodermia localizada; Revisão
\end{abstract}

\begin{abstract}
Localized scleroderma or morphea affects school-aged children, is usually self-limited and a disfiguring condition. Several etiopathogenic factors, investigations and treatment options are described. This article reviews the recent literature and discusses its clinical applications.

Keywords: Child; Childhood; Review; Scleroderma, Localized
\end{abstract}

\section{INTRODUÇÃO}

Esclerodermia é um termo utilizado para descrever um espectro de condições caracterizadas pelo endurecimento e/ou espessamento da pele e pela fibrose dos tecidos envolvidos. É didaticamente dividida nas formas sistêmica e localizada. ${ }^{1,2}$ A forma localizada, também conhecida por morféia, distingue-se pelo envolvimento predominantemente cutâneo, mas com possibilidade de acometimento ocasional dos músculos subjacentes, ao passo que os órgãos internos, geralmente, são poupados.

\section{HISTÓRICO}

As áreas de endurecimento da pele foram descritas primeiramente em 1854, por Addison, que as denominou queloides. O termo morféia foi introduzido por Wilson, originalmente. Em 1868, Fagee fez a diferenciação com queloides e descreveu diversas formas da esclerodermia localizada, incluindo a variante "golpe de sabre."1 Em 1942, Klemper e col. incluíram a esclerodermia no grupo das colagenoses.

\section{EPIDEMIOLOGIA}

A esclerodermia localizada é uma doença rara. O maior estudo populacional foi realizado por Peterson e col. ${ }^{3,4}$ e avaliou a prevalência e a incidência da morféia e de seus subtipos em Minnesota por um período de 33 anos (1960 a 1993). Identificaram-se 82 casos e a incidência anual foi de 2,7 casos por 100.000 habitantes. Exceto para o subtipo linear, no qual não houve predileção por sexo, a relação do acometimento entre homens e mulheres foi de 2,6:1. A média de idade ao diagnóstico foi de 30 anos (as idades dos pacientes variaram de 1 a 82 anos) e, no grupo com forma linear, de 18 anos. Em nenhum dos casos avaliados houve acometimento grave de órgãos internos ou evolução para a forma sistêmica da esclerodermia. Artrites, sinovites, uveíte e contraturas articulares foram mais freqüentes nas categorias de esclerodermia linear e acometimento profundo. As deformidades que acarretaram incapacidade física ocorreram em $11 \%$ dos pacientes, sendo $44 \%$ no grupo de acometimento profundo. A taxa de sobrevida foi semelhante à da população geral. ${ }^{3,4}$

\footnotetext{
Aprovado pelo Conselho Editorial e aceito para publicação em 18.12.08.

* Trabalho realizado no Serviço de Dermatologia do Hospital Universitário de Brasília - Brasília (DF), Brasil.

Conflito de interesse: Nenhum / Conflict of interest: None

Suporte financeiro: Nenhum / Financial funding: None

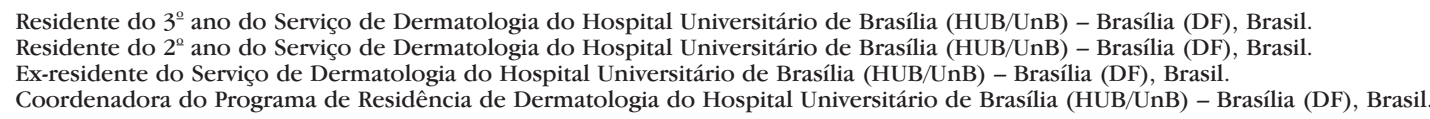




\section{ETIOLOGIA}

A causa da esclerodermia é desconhecida. Já houve relatos de associação com trauma, infecções virais e bacterianas, vacinação e doenças autoimunes. Observou-se a ocorrência de morféia após infecção viral por Epstein-Barr e infecção bacteriana por Borrelia burgdorferi. A detecção de espiroquetas em lesões de morféia, na década de 80 , levantou a possibilidade de que Borrelia burgdorferi fosse um agente etiológico, ${ }^{5,6}$ porém, novos estudos não confirmaram tal associação. ${ }^{7,8}$ Observaram-se lesões da esclerodermia localizada após vacinação com BCG e tríplice viral, ${ }^{9}$ procedimentos cirúrgicos, varicela e traumatismo local. Diante disso, questionou-se se agressões de diversas naturezas poderiam liberar mediadores inflamatórios e citocinas, induzindo fibrose. ${ }^{2} \mathrm{O}$ caráter autoimune desta afecção é sugerido pela presença de autoanticorpos e de doenças autoimunes nos pacientes e familiares, pela deposição de imunoglobulinas e complemento (C3) no exame histopatológico e pela ocorrência de morféia na fase crônica da doença do enxerto versus hospedeiro.

\section{PATOGENIA}

Como em outras doenças autoimunes, postulase que um fator ambiental em um indivíduo geneticamente predisposto ativaria o sistema imunológico com a liberação de citocinas, proliferação dos fibroblastos, produção de colágeno e fibrose. Embora morféia e esclerodermia sistêmica sejam condições distintas, é provável que representem parte do mesmo processo patológico, com três distúrbios básicos ${ }^{2,10,11}$ identificados na patogênese: dano vascular, alteração da imunorregulação e distúrbio do metabolismo do colágeno. Infiltração perivascular e injúria das células endoteliais ocorrem nos estágios iniciais da esclerodermia localizada. Elevação sérica da molécula de adesão presente nos vasos sanguíneos, VCAM-1 (vascular cell adbesion 1) e de E-selectina (receptores encontrados nas células vasculares que mediam as interações iniciais de adesão entre leucócitos intravasculares e o endotélio) são evidência do dano vascular causado pelas células inflamatórias. ${ }^{12}$ Biópsias da pele lesional e não lesional mostram a parede vascular espessada e infiltrada por macrófagos e mastócitos. ${ }^{13}$ Moléculas reguladoras do complemento das células endoteliais parecem estar diminuídas, deixando o endotélio susceptível à ação do complemento autólogo, com consequente injúria endotelial. ${ }^{2} \mathrm{O}$ dano vascular parece levar à liberação de citocinas e à exposição do subendotélio às plaquetas circulantes, provocando a deposição de fibrina e a eventual formação de trombos intravasculares. ${ }^{10}$ Estudos histológicos e imunoistoquímicos sugerem que a fibrose cutânea na esclerodermia inicia-se em torno dos capilares na derme reticular, próxima às células adiposas e ao tecido subcutâneo. ${ }^{14}$ Várias alterações imunológicas ocorrem na esclerodermia. Há diversas evidências sugerindo que a inflamação crônica e persistente tem um importante papel na patogênese de morféia. Demonstrou-se que a extensão do infiltrado linfocítico se correlaciona com a gravidade e a progressão da esclerose cutânea. ${ }^{15}$ No infiltrado cutâneo de células mononucleares predominam os linfócitos T CD4+. A ativação de linfócitos T é evidenciada pela elevação das interleucinas IL-2, IL-4, IL-6 e do receptor da IL-29. A expansão da população dessas células inflamatórias no tecido afetado libera as citocinas e os fatores de crescimento que iniciam e/ou perpetuam o processo de fibrose e dano vascular. Um dos fatores de crescimento que parece desempenhar papel fundamental no processo de fibrose da esclerose é o fator de transformação do crescimento beta (TGF- $\beta$ ). Pequenas quantidades de TGF- $\beta$ agem sobre os fibroblastos, sensibilizando-os e mantendo-os persistentemente ativados por meio de uma sinalização autócrina que estimula, além de outros processos, uma produção adicional de TGF- $\beta{ }^{16,17}$

Além da ativação dos linfócitos T, alterações dos linfócitos B também desempenham importante papel na patogênese da morféia. ${ }^{1,2} \mathrm{~A}$ presença de anticorpos antinucleares (ANA) e de fator reumatoide (FR) também está associada à disfunção das células $\mathrm{B}$.

Um estudo evidenciou que os fibroblastos perivasculares da esclerodermia apresentam aumento do RNA mensageiro responsável pela síntese do colágeno18. Também há aumento da síntese de fibronectina e glicosaminoglicamos pelos fibroblastos. Nas lesões da morféia ocorre aumento dos colágenos tipos I, II, IV. ${ }^{19}$ Essa atividade fibroblástica está alterada pela interação com as células inflamatórias e as citocinas por elas liberadas. As IL-1 e IL-4 estimulam a proliferação fibroblástica e a síntese de colágeno, ao passo que a IL-6, as metaloproteinases da matriz. ${ }^{15}$ Além disso, Lê Roy $^{20}$ e, posteriormente, Jimenez e col. ${ }^{16}$ verificaram que fibroblastos retirados da pele afetada pelas lesões da esclerodermia mantêm uma superprodução de colágeno e de componentes da matriz extracelular in vitro, sugerindo que essas células poderiam ter, também, um padrão de biossíntese alterado.

\section{HISTOPATOLOGIA}

A esclerodermia é caracterizada histologicamente por fibrose e espessamento das fibras de colágeno. ${ }^{2} \mathrm{Na}$ fase inflamatória inicial, ${ }^{1,21}$ particularmente na borda violácea ativa das lesões, os feixes colágenos da derme reticular encontram-se espessados e há um infiltrado inflamatório intersticial e perivascular predominantemente linfocítico, que pode comprometer a gordura subcutânea, com espessamento de trabéculas e substituição por colágeno neoformado. $\mathrm{Na}$ fase 
esclerótica tardia, como visto no centro das lesões, há uma importante redução do infiltrado inflamatório. Os feixes de colágeno na derme reticular tornam-se espessados, agregados, hipocelulares e hiperosinofílicos. Na derme papilar, fibras normais podem ser substituídas por colágeno homogêneo. Vasos sanguíneos são pouco encontrados dentro do colágeno esclerótico, frequentemente têm o lúmen estreito e uma parede fibrótica. ${ }^{1,21}$ As glândulas exócrinas são atróficas, com raros adipócitos ao redor, e são circundadas por colágeno neoformado. Folículos pilosos e glândulas sebáceas estão ausentes.

Nos tipos de morféia linear, segmentar, subcutânea e generalizada, a fáscia e os músculos estriados subjacentes podem estar afetados, sendo que as fibras musculares se apresentam vacuoladas e separadas umas das outras por edema e coleções focais de células inflamatórias. ${ }^{21}$

A epiderme, embora possa se tornar atrófica, é usualmente normal, mesmo nas lesões tardias. Depósitos de IgM e C3 perivascular e na membrana basal são descritos na variante linear da esclerodermia localizada.

\section{MANIFESTAÇÕES CLÍNICAS}

A esclerodermia localizada pode variar desde pequenas placas até doença extensa, com deformidades estéticas e funcionais. ${ }^{22}$ Raramente se observa atrofia das estruturas subjacentes. ${ }^{19}$ A morféia é dez vezes mais comum do que a esclerose sistêmica, é rara entre os 20 e os 50 anos e ocorre, geralmente, na idade escolar. ${ }^{22}$

A doença é, usualmente, autolimitada, com progressão variável com o tipo clínico: mais rápida na forma linear em membros e gradual na forma em placas. O prognóstico é bom: formas superficiais melhoram após três anos, em média. ${ }^{23,24}$ Clinicamente, subdivide-se em cinco formas: em placas, linear, em golpe de sabre, generalizada e panesclerótica. Após a formação de eritema inicial, ocorre a formação de placa de coloração amarelo-brancacenta, elevada ou deprimida, limitada por eritema azul-violáceo (lilac ring). À medida que a infiltração da pele ocorre, observa-se atrofia com perda de fâneros e glândulas sebáceas e hiperpigmentação ou hipopigmentação.

\section{Morféia em placas ou circunscrita}

Trata-se da apresentação clínica mais frequente na população geral. ${ }^{23}$ Apresenta dois subtipos: superficial e profunda. Ocorre no tronco, de modo geral, e acomete, principalmente, a área sobre as cristas ilíacas. Nesta forma clínica, há progressão de placa com anel violáceo para infiltração nacarada sem fâneros e anidrótica. ${ }^{25} \mathrm{~A}$ lesão final são placas circunscritas, enduradas, infiltradas, com bordas mal definidas, com
$2 \mathrm{~cm}$ a $15 \mathrm{~cm}$ de diâmetro, ovais ou redondas. As lesões são mais palpáveis que visíveis. O lilac ring pode ser evidente após meses ou anos. ${ }^{19}$

A lesão pode estender-se mais frequentemente para o tórax, o abdome, as nádegas e os membros inferiores. $\mathrm{O}$ acometimento de face, pescoço e membros superiores é raro (Figura 1). ${ }^{23}$

Morféia gutata é o termo usado para descrever múltiplas lesões escleróticas hipopigmentadas, que podem representar morféia disseminada, as quais devem ser diferenciadas do líquen escleroso e atrófico. $^{23,26} \mathrm{~A}$ coexistência dessas afecções já foi descrita. ${ }^{27}$

A morféia subcutânea localiza-se no tronco superior, nos braços e nas pernas, podendo ser unilateral ou circular nas coxas e nas nádegas. Há acometimento subcutâneo e das camadas profundas da derme, com aspecto cutâneo de peau d'orange e, por vezes, ulceração. A pele torna-se imóvel e pode haver contraturas articulares e artralgias. ${ }^{28}$

\section{Morféia linear dos membros e do tronco}

A esclerodermia linear é o subtipo mais comum em crianças e adolescentes, respondendo por $60 \%$ dos casos, especialmente, em meninas em idade escolar. Clinicamente, observam-se faixas de esclerose lineares que podem estender-se à derme, ao subcutâneo, aos músculos e aos ossos, gerando deformidades (Figura 2). ${ }^{22} \mathrm{~A}$ bilateralidade é incomum. Embora raro, pode ser observado acometimento unilateral da face, do membro superior e da perna - hemiatrofia. ${ }^{23,29}$

\section{Morféia linear da face, em golpe de sabre (en coup de sabre)}

Manifesta-se, geralmente, sob a forma unilateral na face (frontoparietal ou hemifacial), com linha central
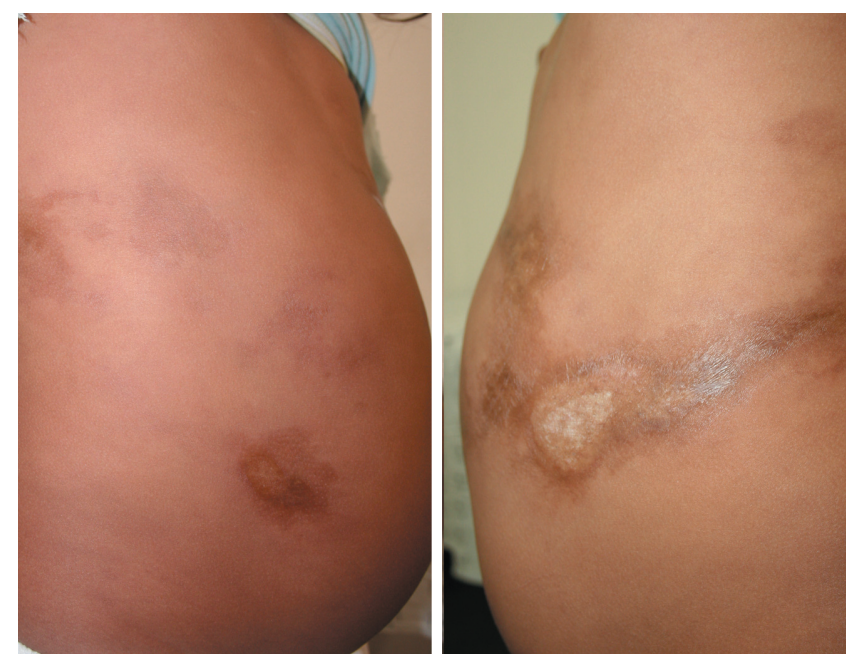

FigurA 1: Placas escleróticas com bordas nítidas, irregulares, hipercrômicas, exibindo atrofia hipopigmentar central em formatos circulares e ovais: morféia em placas em menina de oito anos 
de demarcação. Após rápida fase de eritema e edema, ocorre esclerose e atrofia, com depressão local. ${ }^{25}$ Após o acometimento cutâneo, os tecidos subjacentes são afetados, podendo levar à hemiatrofia da face.

A particularidade da esclerodermia em golpe de sabre reside na possibilidade de complicações do sistema nervoso central (convulsões, cefaléia, hemiparesia ou sintomas focais), dos olhos (uveíte ou vasculites) ou da mandíbula (mau alinhamento e problemas dentários) ${ }^{30,31,32,33}$ Os achados imagenológicos (RNM, SPECT) incluem atrofia ou calcificações corticais. Análise liquórica é compatível com inflamação intracerebral (Figura 3)..$^{34,35}$

Na hemiatrofia facial de Parry-Romberg, ocorrem alterações ósseas, musculares e do tecido subcutâneo, com ou sem esclerose cutânea. Pode coexistir com a morféia em golpe de sabre e sua posição nosológica ainda é questionada. Espectro da esclerodermia linear facial, ou entidade separada, apresenta alterações neuroftalmológicas similares. ${ }^{35,36}$

A ocorrência de epilepsia relaciona-se à presença de lesão cerebral subjacente à esclerose. Achados imagenológicos e histopatológicos evidenciaram processos inflamatórios focais que, em sua maioria, são progressivos $^{37}$

\section{Morféia generalizada}

Condição rara, manifesta-se nos casos de esclerose disseminada sem acometimento sistêmico. Majoritariamente de adultos, é diagnosticada quando placas em número de quatro ou mais se tornam maiores do que $3 \mathrm{~cm}$ e confluem, envolvendo duas das sete principais áreas anatômicas (cabeça-pescoço, membro superior ou inferior esquerdo ou direito, tronco anterior ou posterior).$^{22}$ Inicia-se no tronco e leva a contra-
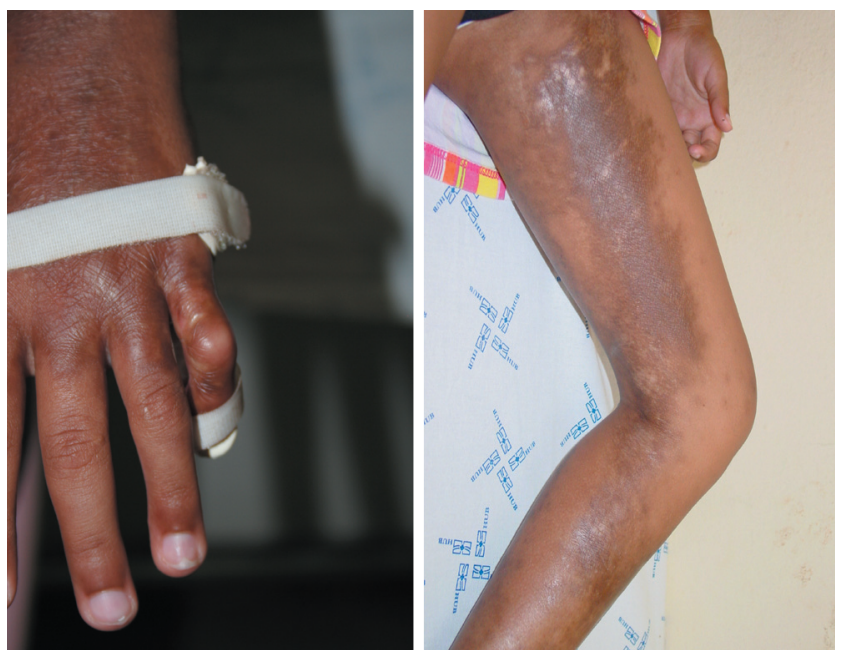

Figura 2: Deformidade em flexão do V quirodáctilo esquerdo e placa esclerótica em perna esquerda - morféia linear em menina de dez anos
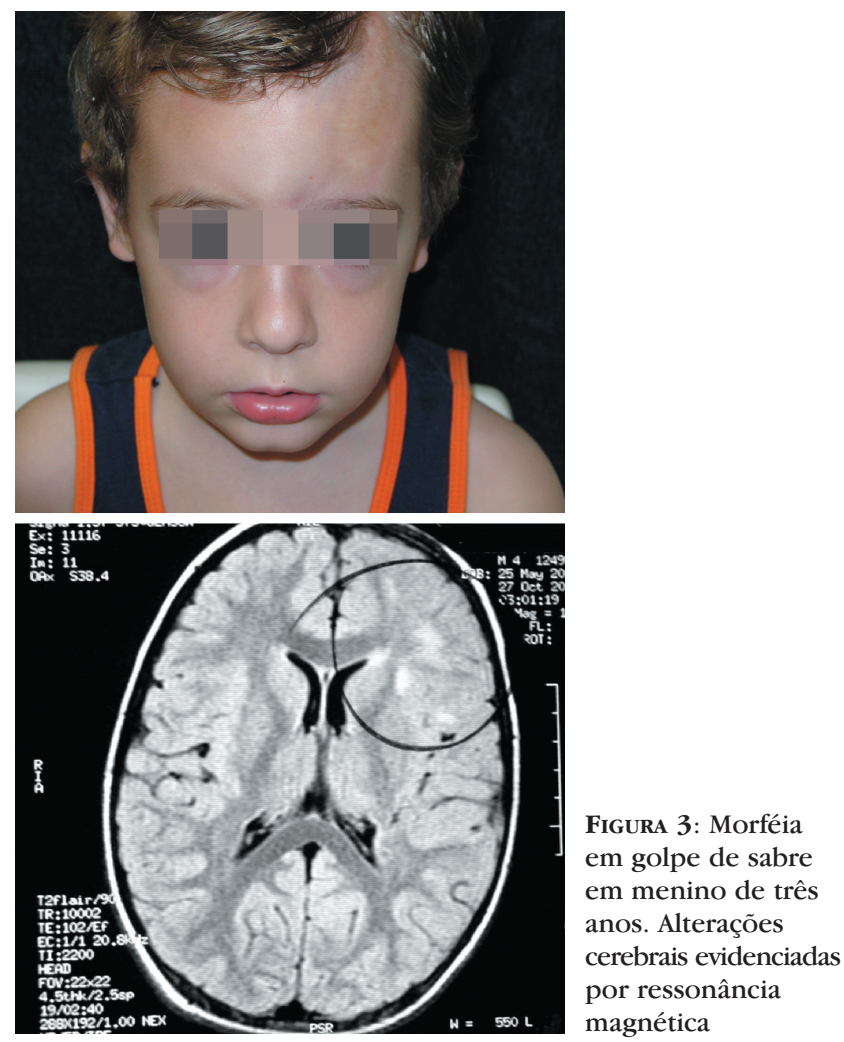

turas e deformidades nos membros. Diferencia-se da esclerose sistêmica por não apresentar fenômeno de Raynauld e não exibir alterações capilaroscópicas. ${ }^{22,23}$ Doença do enxerto versus hospedeiro crônica pode resultar em morféia generalizada.

\section{Morféia panesclerótica}

Trata-se de doença grave, extremamente rara, caracterizada por acometimento de espessura total, generalizado. Envolvimento de articulações gera artralgias e edema articular. Observam-se, comumente, contraturas e retrações dos membros. A presença de ulcerações crônicas já foi descrita em associação a carcinoma espinocelular (Figura 4). ${ }^{35,38,39,40,41}$

Formas combinadas: Pode haver coexistência de formas combinadas, como a linear e em placas, o que é mais frequente em crianças. De fato, metade dos pacientes com a forma linear apresenta, concomitantemente, a forma em placas. Também se veem combinações entre lesões em placas no tronco e linear nos membros ou em golpe de sabre na face. A sobreposição de formas clínicas denota fisiopatologia comum. Outras formas combinadas incluem morféia e líquen escleroso e atrófico, bem como morféia e a atrofoderma de Pasini e Pierini. ${ }^{23}$

Outros achados: Geralmente limitada à pele e ao subcutâneo, a morféia não envolve órgãos internos, mesmo em formas graves. A doença em crianças associa-se, por vezes, a sintomas sistêmicos, entre os 

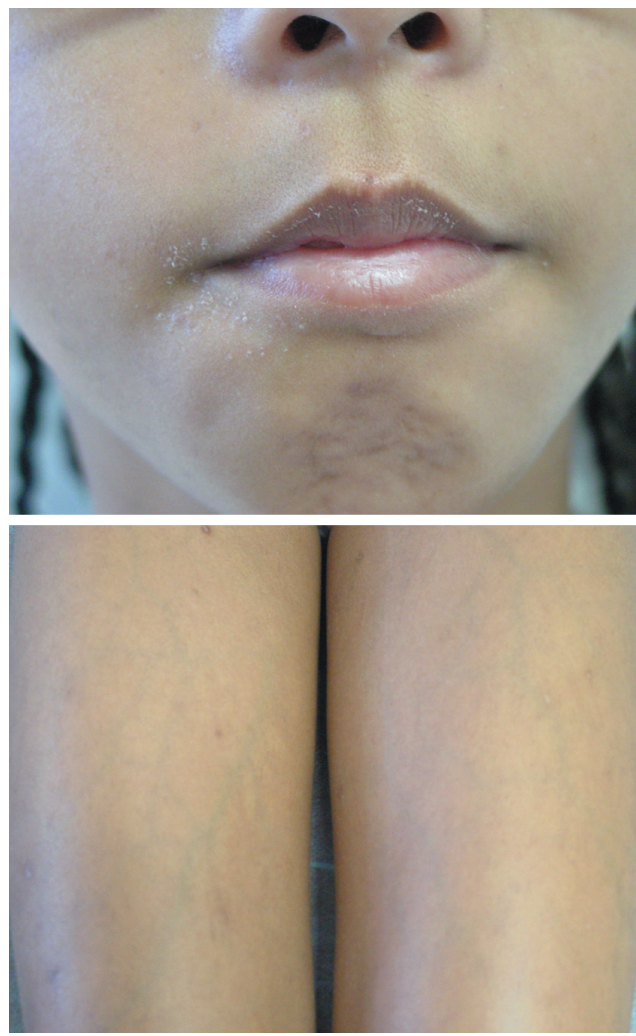

Figura 4:

Morféia

panesclerótica em criança de oito anos

quais, artralgias, sintomas Raynauld-símiles, cólicas abdominais, refluxo gastroesofágico, sintomas neurológicos, defeitos de condução cardíaca, pericardite e dores musculares inespecíficas. ${ }^{19}$ Tais sintomas, em geral, são leves ou assintomáticos, sendo mais comuns em formas cutâneas mais profundas.

A morféia já foi descrita em associação a vitiligo, líquen plano, alopecia areata e colagenoses, como lúpus sistêmico eritematoso, dermatomiosite e artrite reumatoide (Figura 5). ${ }^{42}$

\section{ANORMALIDADES LABORATORIAIS}

Várias anormalidades laboratoriais são encontradas na morféia. Pode-se observar eosinofilia em pacientes com esclerodermia linear ou generalizada e essa condição se correlaciona, freqüentemente, com a extensão da doença. Hipergamaglobulinemia com elevações policlonais de IgM e IgG ocorre em quase 50\% dos pacientes com doença cutânea severa e é mais comum durante a progressão clínica. ${ }^{43}$

Em um estudo internacional com 750 casos de esclerodermia localizada juvenil, 44 reagentes de fase aguda encontraram-se elevados, principalmente, nos casos de morféia profunda. Na forma linear, o VHS mostrou-se elevado em $22,2 \%$ dos casos.

Vários autoanticorpos são encontrados na morféia, $45,46 \mathrm{com}$ altos títulos correlacionando-se com a severidade da doença, incluindo anticorpos antinúcleo (46\%-80\%), anti-ssDNA (50\%) e anti-histona (47\%).
A freqüência do anticorpo antinúcleo (ANA) em crianças com morféia difere de estudo para estudo, variando de $23 \%$ a $73 \%$. Ele é mais comumente encontrado em pacientes com morféia generalizada, seguido pelos casos de esclerodermia linear e morféia localizada. ${ }^{47}$ Altos níveis de anticorpo anti-DNA de hélice simples (anti-ssDNA) foram observados na esclerodermia localizada. Os pacientes com envolvimento muscular tinham níveis maiores desse anticorpo, se comparados aos que não apresentaram comprometimento muscular. Os títulos de anti-ssDNA são bem correlacionados com a atividade da doença. Falanga e col. reportaram alta frequência de anti-ssDNA na morféia generalizada. ${ }^{48}$ Observou-se associação entre antissDNA e contraturas articulares, deformidades ou doença mais extensa, ativa e prolongada. ${ }^{47}$

Em outro estudo, pelo método Elisa, também se verificaram anticorpos anti-histonas em $47 \%$ dos pacientes com esclerodermia localizada e em $87 \%$ dos pacientes com morféia generalizada. A presença dos anticorpos anti-histona teve forte correlação com o número de lesões de morféia e o número de áreas do corpo envolvidas. No caso da morféia generalizada, a sensibilidade é de $87 \%$ e a especificidade, de $74 \%{ }^{48}$

Anticorpos anticardiolipina foram positivos em $46 \%$ dos pacientes com esclerodermia localizada, sendo que $67 \%$ deles apresentavam morféia generalizada e 35\%, esclerodermia linear. Na forma generalizada, a frequência dos anticorpos anticardiolipina IgM (61\%) foi muito maior que a de $\operatorname{IgG}(28 \%){ }^{48}$

Fator reumatoide (FR) pode ser encontrado em $39 \%$ dos pacientes43 e tem correlação significativa com a presença de artrite.$^{44} \mathrm{Na}$ esclerodermia localizada juvenil, FR foi encontrado em 16\% dos pacientes, indicando correlação significativa com a presença de
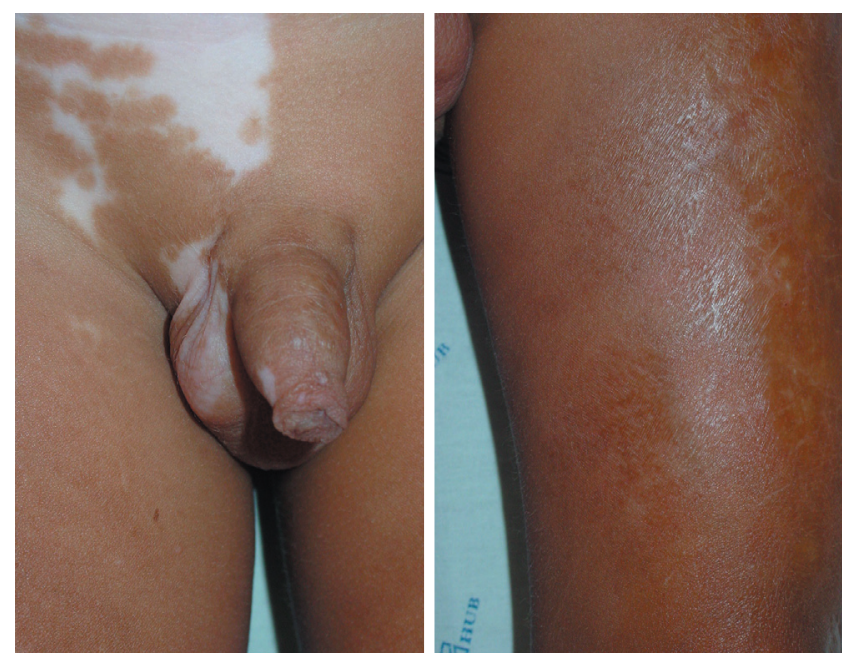

FigurA 5: Associação de morféia em placas e vitiligo em criança de cinco anos de idade 
artrite. Em adultos, sua forma IgM está presente em até $60 \%$ dos pacientes e parece correlacionar-se com a severidade da doença. ${ }^{45,46,48,49}$

Autoanticorpos mais específicos, como Scl-70, anticentrômero, Ro/La e U1RNP, podem preceder o desenvolvimento de doença sistêmica, e os pacientes que apresentam esses marcadores devem ser acompanhados por vários anos. ${ }^{50}$ Pacientes com anti-Scl $70 \mathrm{e}$ anticentrômeros positivos apresentaram sinais ou sintomas de envolvimento orgânico após um seguimento de três a quatro anos. Ainda é incerto se esses anticorpos são marcadores que refletem o componente imunológico da doença ou têm significado prognóstico. ${ }^{44}$

Níveis séricos do fator de necrose tumoral alfa (TNF- $\alpha$ ) e interleucina 13 (IL-13), potentes estimuladores de fibrose, são significativamente elevados em pacientes com esclerodermia localizada, em comparação com indivíduos saudáveis. ${ }^{45}$

\section{TÉCNICAS DE IMAGEM}

O monitoramento da atividade da lesão na esclerodermia linear é, frequentemente, avaliado pela termografia. ${ }^{47,51}$ Ele é capaz de detectar aumento de temperatura nas áreas ativas e em áreas de lesóes novas. ${ }^{52}$ Uma lesão de morféia é considerada termograficamente ativa se aparenta ao observador estar em uma diferença de temperatura de $+0,5^{\circ} \mathrm{C}$ em relação à área adjacente ou ao membro contralateral. ${ }^{53}$

Martini e col., comparando avaliações termográficas com as descrições clínicas das lesões, mostraram que a termografia tinha uma sensibilidade de $92 \%$ e especificidade de $68 \%{ }^{51}$ Esse é um método diagnóstico promissor, quando associado à avaliação clínica, para se determinar a atividade da doença, se aplicado em lesões sem um grau severo de atrofia., ${ }^{1,51,54}$

Estudos radiológicos, especialmente a ressonância nuclear magnética (RNM), são maneiras efetivas de visualizar anormalidades estruturais e documentar a progressão da atrofia do tecido conjuntivo e as alterações ósseas, e são especialmente úteis nas formas linear e em golpe de sabre. Em uma revisão dos achados radiológicos em 23 pacientes, dos cinco com envolvimento de membros inferiores, todos apresentavam discrepâncias no comprimento das pernas, deformidades no remodelamento ósseo e atrofia da gordura subcutânea e dos músculos adjacentes. A RNM é claramente útil na suspeita de comprometimento ocular ou do $\mathrm{SNC}^{45,50,55}$

A ultrassonografia de alta frequência (US) fornece informações sobre a anatomia da pele, detalhando as camadas da derme e do subcutâneo. ${ }^{53}$ A US de 20MHz permite a determinação da profundidade e da extensão da esclerose na área afetada. ${ }^{56}$ Em pacientes pediátricos, pode mostrar a perda de gordura subcutânea e de músculo, aumento do fluxo sanguíneo e aumento da ecogenicidade, por condensação do colágeno na derme. A hiperemia e o aumento de ecogenicidade parecem ser sinais de lesões ativas. ${ }^{57}$ Duas grandes limitações desse recurso são a avaliação operador-dependente e a falta de validação como medida evolutiva em estudos prospectivos. ${ }^{45,57}$

Outras técnicas que têm potencial para fornecer informações são a fluxometria por laser Doppler e a imagem por laser Doppler. ${ }^{53}$ Um estudo recente com 41 crianças mostrou que o fluxo sanguíneo medido por essa técnica era significativamente aumentado nas lesões de esclerodermia localizada clinicamente ativas. Foi encontrada uma sensibilidade de $80 \%$ e uma especificidade de $77 \%$ para detectar as lesões ativas, nos casos de fluxo sanguíneo aumentado em pelo menos $39 \%{ }^{58}$

\section{PROGNÓSTICO}

Em termos de progressão para envolvimento sistêmico, o prognóstico da morféia é geralmente bom. A evolução para esclerodermia sistêmica é rara ( $0,9 \%$ a $5,7 \%$ das coortes). As lesões de morféia raramente coexistem com outras doenças autoimunes sistêmicas, como dermatomiosite, polimiosite, LES, cirrose biliar primária, artrite reumatoide e doença mista do tecido conjuntivo. ${ }^{46}$

Embora a esclerodermia localizada tenha um bom prognóstico, ela pode envolver não só a pele, como também o tecido subcutâneo, músculos, ossos e sinóvia, levando a deformidades e disfunções. ${ }^{59}$ Algumas crianças terão remissões espontâneas e completas, mesmo que a doença, inicialmente, tenha se apresentado severa; outras terão um curso progressivo, debilitante e fatal. ${ }^{55}$

Os tipos de morféia em placa, isolada, disseminada e gutata apresentam, usualmente, um curso moderado e autolimitado. A atividade clínica persiste, em geral, por três a quatro anos. ${ }^{46,53}$

Aproximadamente $20 \%$ dos pacientes com esclerodermia localizada nos membros inferiores desenvolvem atrofia significativa dos tecidos adjacentes, podendo ter como resultado uma discrepância de $1,5 \mathrm{~cm}$ a $7 \mathrm{~cm}$ no comprimento das pernas. Tratamento imediato torna-se imperativo. ${ }^{59} \mathrm{O}$ membro pode se tornar contraído em casos severos e deformidades significativas nas pernas podem se desenvolver em crianças que passaram por uma fase inflamatória prolonga$\mathrm{da}$, conforme observado com a paciente da figura $2 .^{53}$

O tipo golpe de sabre pode evoluir com atrofia óssea, deformidade mandibular, posições anormais dos dentes e dano estético pela hemiatrofia ou deformidade do crânio e SNC. ${ }^{53}$

Infelizmente, os pacientes pediátricos estão sob maior risco de morbidade a longo prazo, devido a alterações de crescimento pelo comprometimento 
ósseo, a assimetrias faciais e nos membros, a distúrbios psicológicos e incapacidades físicas..$^{50,60}$

Diagnóstico diferencial: Algumas síndromes clínicas, que devem ser consideradas, incluem alterações nas unhas e flexuras digitais associadas a condições endocrinológicas (diabetes e tireoidopatias), a erros inatos do metabolismo, como as mucolipidoses e a fenilcetonúria, a escleredema pós-infeccioso e a artropatias. $^{55}$

A diferenciação em relação à esclerodermia sistêmica não é difícil na morféia em placa, mas pode ser dificultada na morféia generalizada e linear. Nesses casos, estudos mais abrangentes podem ser necessários. O fenômeno de Raynauld e o envolvimento de órgãos internos são os achados mais comuns que se devem procurar para se estabelecer essa distinção. Esclerodactilia e pitting ungueal estão raramente presentes nas formas localizadas. Formas profundas de morféia podem ser difíceis de diferençar da paniculite lúpica e de outras paniculites. Estudos histológicos e imunopatológicos podem ser necessários para o estabelecimento dessa diferenciação. ${ }^{61}$

A morféia em placa, no seu estágio inicial, deve ser distinguida do eritema anular, do eritema migrans e da esclerodermia. Também têm sido descritas lesões similares à sarcoidose. Lesões atróficas isoladas da variante em placa podem mostrar similaridade com o atrofoderma após injeção intramuscular de corticosteroides e vitamina $\mathrm{K}$. O tipo gutata se assemelha com o líquen escleroso e atrófico; entretanto, alterações histológicas ajudam na diferenciação. A hemiatrofia facial causada pela variante golpe de sabre é difícil de distinguir da atrofia hemifacial progressiva sem lesões esclerodérmicas, a chamada doença de Parry-Romberg. Questiona-se se ela é uma doença distinta ou uma variante do escleroderma linear, mas muitos concordam que são formas semelhantes e relacionadas do escleroderma craniofacial. ${ }^{50,53}$

Esclerodermia linear nas extremidades, em crianças, deve ser diferençada da fasciíte eosinofílica (síndrome de Shulman). Entretanto, essa é uma síndrome muito rara na infância. ${ }^{53}$

Tratamento: Devido à etiologia desconhecida, nenhum tratamento específico para a doença está disponível. Alguns medicamentos tópicos e sistêmicos, aliados a fisioterapia, podem ser efetivos em limitar a progressão da doença. ${ }^{53} \mathrm{O}$ objetivo da terapia é impedir esta de maneira precoce, de modo a prevenir o desenvolvimento de complicações funcionais e estéticas. ${ }^{1,54}$ As escolhas terapêuticas devem ser feitas após avaliação da extensão e severidade da doença, bem como da taxa de progressão da mesma, e depois de uma discussão a respeito dos potenciais riscos e benefícios das opções terapêuticas tanto para os pais como para os filhos. ${ }^{55}$
As drogas usadas são, em geral, voltadas à supressão da inflamação e das alterações do colágeno. Numerosos tratamentos, como penicilamina, antimaláricos, retinoides, calcitriol, calcipotriol, imiquimod, ciclosporina, interferon e irradiação com UVA, têm sido usados no cuidado com a esclerodermia localizada, com graus variáveis de sucesso e frequente limitação de efeitos nas formas linear e profunda. Corticosteroides e baixas doses de metotrexato (MTX) têm sido repetidamente relatados como tratamentos benéficos para a esclerodermia linear; em crianças, o MTX tem sido o recurso mais regularmente utilizado nos últimos cinco anos. ${ }^{1,44}$

Algumas vezes, resultados estimuladores com corticosteroides tópicos potentes são obtidos nas fases precoces da doença, especialmente, na morféia em placa. ${ }^{47,53}$ No caso de lesões únicas em placa, os pacientes devem ser informados do caráter benigno e geralmente autolimitado da doença. Podem-se usar esteroides tópicos ou oclusivos para aumentar a absorção. Triancinolona intralesional tem sido eficaz em diminuir a progressão quando injetada nas margens das lesões. Entretanto, isso aumenta o risco de induzir necrose ou lipoatrofia. A hidratação diária é benéfica. Em lesões pruriginosas, pode-se recorrer à capsaicina tópica. $^{56}$

Quando a lesão aumenta rapidamente ou envolve funcionalmente áreas importantes, a terapia sistêmica deve ser indicada. Nesses casos, corticosteroides orais são a primeira escolha. As indicações para corticoterapia oral na esclerodermia localizada são: a) achados inflamatórios clínicos exuberantes, com rápida progressão da lesão; b) prejuízo funcional ou previsão de prejuízo funcional; c) possibilidade de comprometimento do crescimento; d) lesão muscular e altos níveis de anticorpos anti-ss DNA. ${ }^{47}$

Weibel e col. publicaram o maior estudo retrospectivo envolvendo séries de crianças com esclerodermia localizada tratadas com corticosteroides e MTX. Todos os pacientes melhoraram significativamente com o tratamento e nenhum deles o abandonou em razão de efeitos adversos. ${ }^{1}$

A prática consiste no seguinte: terapia de indução com metilprednisolona $30 \mathrm{mg} / \mathrm{kg} / \mathrm{dia}$ (máxima dose de $500 \mathrm{mg} / \mathrm{dia}$ ), administrada em infusão diária por três dias e repetida por mais três dias na semana seguinte. Administra-se prednisona oral após o primeiro curso de metilprednisolona, na dose inicial recomendada de $0,5-1,0 \mathrm{mg} / \mathrm{kg} / \mathrm{dia}$, por pelo menos quatro semanas, e, a seguir, reduz-se a dose gradualmente, por um período total de três a seis meses. O tratamento de manutenção é feito com o MTX, iniciado após o segundo pulso de metilprednisolona. A dose inicial é de $10 \mathrm{mg} / \mathrm{m}^{2}$ de superfície corporal por semana, que pode ser administrada por via oral ou 
subcutânea. Quando a atividade da doença está controlada, a dose pode ser reduzida, mas a droga deve ser continuada por pelo menos um ano após a doença se tornar inativa. ${ }^{53} \mathrm{O}$ tratamento de manutenção deve ser mantido por um a três anos e os pacientes devem ser regularmente acompanhados, após o fim do tratamento, por pelo menos cinco anos. Sugere-se fazer um monitoramento, durante todo o período de tratamento, com exames de sangue a cada quatro a seis semanas (hemograma completo, eletrólitos, uréia, creatinina, testes de função hepática, em particular, ALT, peso e altura) e, enquanto perdurar o uso de corticosteroides, acompanhar a pressão arterial e urinálise. . $33,54,55^{-1}$

Uziel e col. acreditam que os corticosteroides são agentes de indução, que reduzem o componente inflamatório da doença, e que o MTX age na inflamação crônica e na fibrose..$^{62} \mathrm{O}$ mecanismo pelo qual as baixas doses de MTX melhoram a fibrose da pele ainda não é totalmente entendido. Ele pode agir diretamente nos fibroblastos ou a melhora da pele se deve a seu efeito anti-inflamatório. ${ }^{1,54}$ Parece agir com base na inibição de várias citocinas (IL-2, 4, 6 e 8), que se mostraram aumentadas na esclerodermia localizada e se correlacionaram com o grau de esclerose cutânea. ${ }^{63}$

A ciclosporina é um metabólito fúngico com atividade imunossupressora potente, afetando tanto a imunidade celular quanto a humoral. Tem mostrado ação efetiva no tratamento da esclerose sistêmica, assim como da morféia incapacitante. Muitos autores notaram, em seus pacientes, uma resposta à ciclosporina mais óbvia nas lesões de pele do que no envolvimento sistêmico. A ciclosporina inibe seletivamente a liberação de IL-2 dos linfócitos ativados. O benefício clínico do seu uso na esclerose sistêmica foi subestimado em razão de seus efeitos colaterais, principalmente, danos renais e hipertensão, provavelmente, devido ao uso de doses mais elevadas do que as usadas para doenças de pele. Atualmente, considera-se a ciclosporina bem tolerada por crianças desde que adequadamente monitorizada, podendo constituir uma importante ferramenta no armamento terapêutico por causa de seu rápido início de ação. ${ }^{59}$

A presença de receptores cutâneos para o calcitriol sugere que a pele não seja apenas órgão produtor desse hormônio, como também órgão-alvo de sua ação. O calcitriol possui ações variadas no escleroderma, como: efeitos imunomodulatórios nas funções de linfócitos e monócitos/macrófagos, inibição da proliferação de fibroblastos dose-dependente ou efeitos diretos na regulação da expressão do gene do colágeno. Elst e col.64 mostraram, apesar do número limitado de pacientes (sete crianças), que o calcitriol sistêmico pode ser um agente terapêutico eficaz na esclerodermia localizada na infância. Observou-se melhora na rigidez da pele e na mobilidade articular em seis crianças, após três meses de tratamento. Sugere-se administrar o calcitriol por pelo menos esse período em crianças com esclerodermia linear, antes de se introduzir terapia mais agressiva. Se se notar melhora, o tratamento deverá ser mantido por seis a nove meses. ${ }^{64,65}$

Em um estudo com 12 pacientes de 12 a 38 anos, portadores de morféia em atividade ou esclerodermia linear, confirmada histologicamente, aplicouse nas placas loção de calcipotrieno a $0,005 \%$ sob oclusão duas vezes ao dia por três meses, observandose melhora significativa e nenhum efeito colateral. ${ }^{66}$

Desde a metade da década de 90 , a fototerapia com radiação ultravioleta tem sido usada para tratar pacientes com esclerodermia localizada e envolvimento cutâneo exclusivo. Os estudos que utilizaram tratamento com UVA1 em pequenas, médias e altas doses, com ou sem psoralenos (PUVA), mostram efeitos clínicos benéficos, embora altas doses pareçam ser mais eficazes. O número de sessões semanais variou de três a cinco e o total de sessões esteve entre 20 e 40. Existe uma variedade de mecanismos de ação pelos quais a fototerapia com ultravioleta pode agir, incluindo o aumento na atividade da colagenase-metaloproteinase-1 da matriz, aumento na expressão de IFN- $\gamma$ e diminuição do TGF- $\beta$. Apesar de sua segurança relativa, existem preocupações quanto à possível malignidade, especialmente em crianças. ${ }^{45}$

Kreuter e col. realizaram um ensaio clínico controlado e randomizado em que compararam a terapia com radiação UVA1 em baixas e médias doses e UVB narrow-band em 64 pacientes com esclerodermia localizada limitada à pele e ao tecido subcutâneo. $\mathrm{O}$ tratamento foi administrado cinco vezes semanais durante oito semanas. Cada tipo de tratamento foi eficaz, embora UVA1 em dose média tenha sido estatisticamente superior que UVB. Observou-se uma significativa melhora histológica somente no tratamento com UVB. Ultrassonografia mostrou uma redução na espessura da pele e um aumento na densidade da derme apenas com dose média de UVA1. ${ }^{67}$

A produção de colagenase pela UVA é dosedependente e diretamente proporcional à quantidade de UVA maior que $60 \mathrm{~J} / \mathrm{cm}^{2}$. Entretanto, uma resposta satisfatória foi encontrada com doses baixas $\left(20 \mathrm{~J} / \mathrm{cm}^{2}\right)$ precocemente no tratamento. Os resultados do estudo de El-Mofty e col. mostram uma resposta clínica satisfatória alcançada com baixas doses de UVA $\left(5 \mathrm{~J} / \mathrm{cm}^{2} /\right.$ sessão, $10 \mathrm{~J} / \mathrm{cm}^{2} /$ sessão e $20 \mathrm{~J} / \mathrm{cm}^{2} /$ sessão), num total de 20 sessões. $^{68}$

Esse mesmo pesquisador participou de outro estudo realizado para demonstrar a eficácia da terapia com UVA em baixas doses, em que 12 pacientes com morféia foram expostos à radiação UVA na dose de 
$20 \mathrm{~J} / \mathrm{cm}^{2}$, três vezes por semana, durante 20 sessões. Algumas lesões em placas foram cobertas para servirem como controle. A eficácia da terapia foi julgada clinicamente e por amostras de biópsias. Todos os pacientes apresentaram notável amolecimento das lesões, confirmado ao exame físico. Uma redução significativa da concentração média de colágeno foi detectada nas placas expostas à UVA, enquanto que, nas placas cobertas, a diferença não foi estatisticamente significante. Os autores concluíram que a fototerapia com UVA em doses baixas é uma modalidade terapêutica bastante eficaz e segura para a esclerodermia localizada. ${ }^{69}$

A fotoquimioterapia com psoralenos e UVA (PUVA) leva à apoptose das células T que infiltram a pele e tem mostrado, por muitos anos, efeito altamente benéfico em doenças como psoríase, dermatite atópica e linfoma cutâneo de células T. Embora estudos controlados não tenham sido publicados, ótimos resultados têm sido descritos com o tratamento com PUVA em pacientes portadores de esclerodermia localizada. ${ }^{70}$

O estudo de Pasic e col. relatou o tratamento de seis crianças portadoras de esclerodermia localizada por meio de fotoquimioterapia com banho de PUVA, com bons resultados em termos do amolecimento das placas escleróticas. Os resultados foram alcançados com um pequeno número de sessões (média de 25, variando de 14 a 39). ${ }^{71}$ Após a radiação UVA, pode-se detectar um aumento na expressão da colagenase nos fibroblastos da derme. Especula-se que a radiação UVA pode suprimir a síntese de colágeno e induzir a atividade da colagenase, resultando na melhora clínica das placas escleróticas. ${ }^{72}$

Grundmann e col. mostraram que o tratamento tópico com PUVA induziu melhora significativa em quatro pacientes, tratados quatro vezes por semana, num total de 30 sessões. A melhora foi demonstrada tanto clinicamente como pela ultrassonografia e análise histopatológica. ${ }^{73}$

Em um ensaio clínico com sete pacientes ao longo de três meses, houve benefício nas lesões tratadas com tacrolimo tópico oclusivo por $12 \mathrm{~h}$ à noite, com amolecimento e redução do eritema inflamatório em todos os pacientes em um mês de tratamento. Porém, estudos duplo-cego controlados são necessários para confirmar seu uso. $\mathrm{O}$ mecanismo pelo qual o tacrolimo induz à regressão das lesões esclerodérmicas não está claro. É provável que exerça função pelo seu efeito imunomodulador e anti-inflamatório, ligado à inibição da ativação dos linfócitos $\mathrm{T}$ e à redução da produção de citocinas inflamatórias. ${ }^{74}$

Imiquimod é um imunomodulador que interfere na ação de várias citocinas, incluindo IFN- $\alpha$ e $\gamma$, capazes de inibir a produção de colágeno nos fibroblastos. ${ }^{45}$ Dytoc e col. relataram um estudo com 12 pacientes com diagnóstico histológico de morféia, tratados com imiquimod, inicialmente, três vezes por semana, seguidas por aplicações diárias. Onze dos 12 pacientes mostraram $29 \%$ a $60 \%$ de melhora em três meses e sete dos 12 pacientes avaliados em seis meses mostraram de $38 \%$ a $100 \%$ de melhora. A análise histológica de quatro pacientes mostrou $4 \%$ a $33 \%$ de melhora na espessura da pele. Os efeitos colaterais foram mínimos e limitados à irritação local, que se resolveu com a redução da frequência de uso do medicamento. Porém, esse foi um estudo não controlado, necessitando de novas investigações para se comprovar seu benefício. ${ }^{75}$

Kreuter e col., bem como Christen-Zaech e col., concluem que a terapia combinada de MTX com corticoide deveria ser considerada como primeira linha de tratamento na esclerodermia localizada severa, que também afeta estruturas extracutâneas como tecido adiposo, músculo, fáscia e/ou ossos. Entretanto, pacientes portadores de morféia em placa, que, usualmente, afeta apenas a derme, não se beneficiam tanto dessa opção terapêutica, considerando-se seus potenciais efeitos colaterais. Esses pacientes, em geral, respondem bem ao tratamento tópico com corticosteroides, análogos da vitamina D3 e/ou fototerapia. A terapia com PUVA deveria ser considerada nos estágios iniciais de inflamação, enquanto a fototerapia com UVA1 é mais benéfica no estágio fibrótico da morféia. ${ }^{76,77}$

Fisioterapia regular é essencial, especialmente, nos casos de morféia linear, para prevenir o desenvolvimento de contraturas. No caso de crianças com encurtamento de pernas, cirurgias ortopédicas podem ser necessárias. ${ }^{52,53}$

Se a criança tem uma doença moderada e, provavelmente, autolimitada, como pequenas placas de esclerodermia linear que não atingem as articulações, observá-la sem intervenções médicas, apenas administrando fisioterapia, pode ser apropriado. Saber quando parar as medicações, uma vez iniciadas, também é um desafio para o médico assistente. Grosso modo, quando não há mais progressão das lesões por seis meses, os medicamentos podem ser lentamente retirados e os marcadores de doença ativa (reagentes de fase aguda, alterações nos exames de imagem) devem ser monitorados. Cirurgia plástica ou técnicas de preenchimento para esclerodermia facial são, normalmente, consideradas, especialmente nos adolescentes, que têm maior percepção de sua aparência. Em geral, se a doença aparenta ter se estabilizado por seis a 12 meses e técnicas médicas e fisioterápicas não obtiveram sucesso, a intervenção cirúrgica deve ser levada em consideração, com atenção especial para o suprimento sanguíneo da região. ${ }^{50,55}$

Em conclusão, a morféia em crianças ocorre 
mais frequentemente em placas; no entanto, causa pouca morbidade. Por outro lado, as formas lineares apresentam, potencialmente, mais risco de incapacidades físicas - como na apresentação segmentar em membros -, ou, ainda, comprometimento neurológico grave - como no golpe de sabre. Essas incapacidades têm alto impacto na vida dos pacientes, inclusive, com dano psicológico importante.

\section{REFERÊNCIAS}

1. Weibel L, Sampaio MC, Visentin MT, Howell KJ, Woo P, Harper JI. Evaluation of methotrexate and corticosteroids for the treatment of localized scleroderma (morphoea) in children. Br J Dermatol. 2006;155:1013-20.

2. Vierra E, Cunningham BB. Morphea and Localized Scleroderma in Children. Semin Cutan Med Surg. 1999; 18:210-25.

3. Peterson LS, Nelson AM, Su WP, Mason T, O'Fallon WM, Gabriel SE. The epidemiology of morphea (localized scleroderma) in Olmsted County 1960-1993. J Rheumatol. 1997;24:73-80.

4. Mayes MD. Classification and epidemiology of Scleroderma. Semin Cutan Med Surg. 1998;17:22-6.

5. Aberer E, Neumann R, Stanek G. Is localised scleroderma a Borrelia infection? Lancet. 1985;11:278.

6. Aberer E, Stanek G, Ertl M, Neumann R. Evidence for spirochetal origin of circumscribed scleroderma (morphea). Acta Derm Venereol. 1987;67:225-31.

7. Buechner AS, Winkelmann RK, Lautenschlager S, Gilli L, Rufli T. Localized scleroderma associated with Borrelia burgdorferi infection. Clinical, histologic, and immunohistochemical observations. J Am Acad Dermatol. 1993;29(Pt 1):190-6.

8. Wienecke R, Schlüpen EM, Zöchling N, Neubert U, Meurer M, Volkenandt M. No evidence for Borrelia burgdorferi-specific DNA in lesions of localized scleroderma. J Invest Dermatol. 1995;104:23-6.

9. Murray KJ, Laxer RM. Scleroderma in children and adolescents. Rheum Dis Clin North Am. 2002;28:603-24.

10. Jimenez Sa, Derk CT. Following the molecular pathways toward an understanding of the pathogenesis of systemic sclerosis. Ann Intern Med. 2004;140:37-50.
O acompanhamento dos pacientes acometidos não pode prescindir da rápida detecção de novas lesões ou sinais de atividade inflamatória em lesões antigas. O tratamento deve objetivar a melhora das lesões, a prevenção de incapacidades, a reabilitação e a melhora estética.

11. Amento EP. Immunologic abnormalities in scleroderma. Semin Cutan Med Surg. 1998;17:18-21.

12. Yamane K, Ihn H, Kubo M, Yazawa N, Kikuchi K, Soma Y, et al. Increased serum levels of soluble vascular cell adhesion molecule 1 and E-selectin in patients with localized scleroderma. J Rheumatol. 2000;27:1917-21.

13. Liu B, Connolly MK. The pathogenesis of cutaneous fibrosis. Semin Cutan Med Surg. 1998;17:3-11.

14. Kobayasi T, Seup J. Vascular changes in morphea. Acta Derm Venereol. 1985;65:116-20.

15. Roumm AD, Whiteside TL, Medsger TA et al. Lymphocytes in the skin of patients with progressive sclerosis. Quantification, subtyping and clinical correlations. Arthitis Reum. 1984;27:644-53.

16. Jimenez SA, Hitraya E, Varga J. Patogénesis of scleroderma: collagen. Rheum Dis Clin North AM. 1996;22:647-74.

17. Roberts AB. Molecular and cell biology of TGF-beta. Miner Electrolyte Metab. 1998;24:11-9

18. Peltonen J, Kähäri L, Uitto J, Jimenez SA. Increased expression of type VI collagen genes in systemic sclerosis. Arthritis Rheum. 1990;33:1829-35.

19. Yu B, Eisen A. Scleroderma. In: Freedburg IM, Eisen AZ, Wolff $\mathrm{K}$, et al. Fitzpatrick's Dermatology in General Medicine. 6th ed. New York: McGraw-Hill Internatinal Book Co., 2003. p.1709-18.

20. Leroy EC. Connective tissue synthesis by scleroderma skin fibroblasts in cell culture. J Exp Med. 1972;135:1351-62.

21. Jaworsky C. Connective tissue diseases. In: Elder D, Elenistsas R, Johnson JR B, Murphy GF. Lever's histopathology of the skin. Philadelphia: Lippincott Willians and Wikins; 2005. p. 310-15. 
22. Laxer RM, Zulian F. Localized scleroderma. Curr Opin Rheumatol. 2006;18:606-13. Review.

23. Bono W, Dupin N. [Localized scleroderma (morphea)]. Presse Med. 2006;35(Pt 2):1923-8. French.

24. Guidolin F, Esmanhotto L, Magro CE, Silva MB, Skare TL. Prevalência de achados cutâneos em portadores de esclerose sistêmica -Experiência de um hospital universitário. An Bras Dermatol. 2005;80:481-6.

25. Krafchik BR.Localized cutaneous scleroderma. Semin Dermatol. 1992;11:65-72.

26. Black CM. Prognosis and management of scleroderma and scleroderma-like disorders in children. Clin Exp Rheumatol. 1994;12 Suppl 10:S75-81. Review.

27. Uitto J, Santa Cruz DJ, Bauer EA, Eisen AZ. Morphea and lichen sclerosus et atrophicus. Clinical and histopathologic studies in patients with combined features. J Am Acad Dermatol. 1980;3:271-9.

28. Whittaker SJ, Smith NP, Jones RR. Solitary morphoea profunda. Br J Dermatol. 1989;120:431-40.

29. Kornreich HK, King KK, Bernstein BH, Singsen BH, Hanson V. Scleroderma in childhood. Arthritis Rheum. 1977;20(2 Suppl):343-50

30. Goldenstein-Schainberg C, Pereira RM, Gusukuma MC, Messina WC, Cossermelli W. Childhood linear scleroderma "en coup de sabre" with uveitis. J Pediatr. 1990;117:581-4.

31. Muchnick RS, Aston SJ, Rees TD. Ocular manifestations and treatment of hemifacial atrophy. Am J Ophthalmol. 1979;88:889-97.

32. Serup J, Alsbirk PH. Localized scleroderma 'en coup de sabre' and iridopalpebral atrophy at the same line. Acta Derm Venereol. 1983;63:75-7.

33. Holl-Wieden A, Klink T, Klink J, Warmuth-Metz M, Girschick HJ. Linear scleroderma 'en coup de sabre' associated with cerebral and ocular vasculitis. Scand J Rheumatol. 2006;35:402-4.

34. Stone J, Franks AJ, Guthrie JA, Johnson MH. Scleroderma 'en coup de sabre': pathological evidence of intracerebral inflammation. J Neurol Neurosurg Psychiatry. 2001;70:382-5.

35. Blaszczyk M, Królicki L, Krasu M, Glinska O, Jablonska S. Progressive facial hemiatrophy: central nervous system involvement and relationship with scleroderma en coup de sabre. J Rheumatol. 2003;30:1997-2004.

36. Tollefson MM, Witman PM. En coup de sabre morphea and Parry-Romberg syndrome: a retrospective review of 54 patients. J Am Acad Dermatol. 2007;56:257-63.

37. Appenzeller S, Montenegro MA, Dertkigil SS, SampaioBarros PD, Marques-Neto JF, Samara AM, Andermann F, Cendes F. Neuroimaging findings in scleroderma en coup de sabre. Neurology. 2004;62:1585-9.

38. Parodi PC, Riberti C, Draganic Stinco D, Patrone P, Stinco G. Squamous cell carcinoma arising in a patient with long-standing pansclerotic morphea. Br J Dermatol. 2001; 144:417-9.

39. Wollina U, Buslau M, Weyers W. Squamous cell carcinoma in pansclerotic morphea of childhood. Pediatr Dermatol 2002;19:151-4.

40. Padilha-Goncalves A. Classificação didática das formas clínicas da esclerodermia. An Bras Dermatol. 1993;68:346-350.
41. Maragh SH, Davis MDP, Bruce AJ, Nelson AM. Disabling pansclerotic morphea: clinical presentation in two adults. J Am Acad Dermatol. 2005;53:S115-9.

42. Pereira LB, Gómez RS, Silva CMR, Santos WF. Você conhece esta síndrome? Síndrome de Parry-Romberg. An Bras Dermatol. 2007;82:90-3.

43. Tuffanelli DL. Localized scleroderma. Semin Cutan Med Surg. 1998; 17:27-33.

44. Zulian F, Athreya BH, Laxer R, Nelson AM, Feitosa de Oliveira SK, Punaro MG, et al. Juvenile scleroderma Working Group of the Pediatric Rheumatology European Society (PRES). Juvenile localized scleroderma: clinical and epidemiological features in 750 children. An international study. Rheumatology (Oxford). 2006; 45:614-20.

45. Laxera RM, Zulianb F. Localized scleroderma. Curr Opin Rheumatol. 2006;18:606-13.

46. Chung L, Lin J, Furst DE, Fiorentino D. Systemic and localized scleroderma. Clin Dermatol. 2006;24:374-92.

47. Sugiura K, Muro Y, Tomita Y. A case of a childhood linear scleroderma with limb asymmetry. Mod Rheumatol. 2004;14:254-6.

48. Takehara K, Sato S. Localized scleroderma is an autoimmune disorder. Rheumatology (Oxford). 2005;44:274-9.

49. Mimura Y, Ihn H, Jinnin M, Asano Y, Yamane K, Tamaki $\mathrm{K}$. Rheumatoid factor isotypes in localized scleroderma. Clin Exp Dermatol. 2005;30:405-8.

50. Holland KE, Steffes B, Nocton JJ, Schwabe MJ, Jacobson RD, Drolet BA. Linear scleroderma en coup de sabre with associated neurologic abnormalities. Pediatrics. 2006;117:e132-6.

51. Martini G, Murray KJ, Howell KJ, Harper J, Atherton D, Woo P, Zulian F, Black CM. Juvenile-onset localized scleroderma activity detection by infrared thermography. Rheumatology (Oxford). 2002;41:1178-82.

52. Uziel Y, Krafchik BR, Silverman ED, Thorner PS, Laxer RM. Localized scleroderma in childhood: a report of 30 cases. Semin Arthritis Rheum. 1994;23:328-40.

53. Sampaio C, Visentin MT, Howell K, Woo P, Harper J. Morphoea. In: Harper J, Oranje A, Prose N. Textbook of Pediatric Dermatology. 2nd ed. Oxford: Blackwell Publishing; 2006. p.2020-9. v. 2.

54. Fitch PG, Rettig P, Burnham JM, Finkel TH, Yan AC, Akin E, Cron RQ. Treatment of pediatric localized scleroderma with methotrexate. J Rheumatol. 2006;33:609-14.

55. Emery H. Pediatric scleroderma. Semin Cutan Med Surg. 1998; 17:41-47.

56. Hunzelmann N, Scharffetter Kochanek K, Hager C, Krieg T. Management of localized scleroderma. Semin Cutan Med Surg. 1998;17:34-40.

57. Li SC, Liebling MS, Haines KA. Ultrasonography is a sensitive tool for monitoring localized scleroderma. Rheumatology (Oxford). 2007;46:1316-9.

58. Weibel L, Howell KJ, Visentin MT, Rudiger A, Denton CP, Zulian F, et al. Laser Doppler flowmetry for assessing localized scleroderma in children. Arthritis Rheum. 2007;56:3489-95.

59. Strauss RM, Bhushan M, Goodfield MJ. Good response of linear scleroderma in a child to ciclosporin. $\mathrm{Br} \mathrm{J}$ Dermatol. 2004;150:790-2. 
60. Hawk A, English JC 3rd. Localized and systemic scleroderma. Semin Cutan Med Surg. 2001;20:27-37.

61. Liu B, Connolly MK. The Pathogenesis of cutaneous fibrosis. Semin Cutan Med Surg. 1988;17:3-11.

62. Uziel Y, Feldman BM, Krafchik BR, Yeung RS, Laxer RM. Methotrexate and corticosteroid therapy for pediatric localized scleroderma. J Pediatr. 2000;136:91-5.

63. Kreuter A, Gambichler T, Breuckmann F, Rotterdam S, Freitag M, Stuecker M, et al. Pulsed high-dose corticosteroids combined with low-dose methotrexate in severe localized scleroderma. Arch Dermatol. 2005;141:847-52.

64. Elst EF, Van Suijlekom-Smit LW, Oranje AP. Treatment of linear scleroderma with oral 1,25-dihydroxyvitamin D3 (calcitriol) in seven children. Pediatr Dermatol. 1999; 16:53-8.

65. Dervis E, Dervis E. Progressive hemifacial atrophy with linear scleroderma. Pediatr Dermatol. 2005;22:436-9.

66. Cunningham BB, Landells ID, Langman C, Sailer DE, Paller AS. Topical calcipotriene for morphea/linear scleroderma. J Am Acad Dermatol. 1998;39(Pt 1):211-5.

67. Kreuter A, Hyun J, Stucker M, Sommer A, Altmeyer P, Gambichler T. A randomized controlled study of low-dose UVA1, medium-dose UVA1, and narrowband UVB phototherapy in the treatment of localized scleroderma. J Am Acad Dermatol. 2006;54:440-7.

68. El-Mofty M, Mostafa W, El-Darouty M, Bosseila M, Nada $\mathrm{H}$, Yousef $\mathrm{R}$, et al. Different low doses of broad-band UVA in the treatment of morphea and systemic sclerosis. Photodermatol Photoimmunol Photomed. 2004;20:148-56.

69. El-Mofty M, Zaher H, Bosseila M, Yousef R, Saad B. Lowdose broad-band UVA in morphea using a new method for evaluation. Photodermatol Photoimmunol Photomed. 2000;16:43-9.

70. De Rie MA, Bos JD. Photochemotherapy for systemic and localized scleroderma. J Am Acad Dermatol. 2000;43:725-6.
71. Pasiç A, Ceoviç R, Lipozenciç J, Husar K, Susiç SM, Skerlev M, et al. Phototherapy in pediatric patients. Pediatr Dermatol. 2003;20:71-7.

72. Kreuter A, Gambichler T, Avermaete A, Jansen T, Hoffmann M, Hoffmann K, et al. Combined treatment with calcipotriol ointment and low-dose ultraviolet A1 phototherapy in childhood morphea. Pediatr Dermatol. 2001; 18:241-5.

73. Grundmann-Kollmann M, Ochsendorf F, Zollner TM, Spieth K, Sachsenberg-Studer E, Kaufmann R, et al. PUVA-cream photochemotherapy for the treatment of localized scleroderma. J Am Acad Dermatol. 2000; 43:675-8.

74. Mancuso G, Berdondini RM. Localized scleroderma: response to occlusive treatment with tacrolimus ointment. Br J Dermatol. 2005;152:180-2.

75. Dytoc M, Ting PT, Man J, Sawyer D, Fiorillo L. First case series on the use of imiquimod for morphoea. $\mathrm{Br} \mathrm{J}$ Dermatol. 2005;153:815-20.

76. Kreuter A, Altmeyer P, Gambichler T. Treatment of localized scleroderma depends on the clinical subtype. Br J Dermatol. 2007;156:1363-5.

77. Christen-Zaech S, Hakim MD, Afsar FS, Paller AS. Pediatric morphea (localized scleroderma): review of 136 patients. J Am Acad Dermatol. 2008;59:385-96.

\author{
ENDEREÇO PARA CORRESPONDÊNCIA / MAILING ADDRESS: \\ Pedro Zancanaro \\ SHIS QI 11 Bloco O Sala 16A Lago Sul \\ 71600700 Belo Horizonte - MG \\ Tel./fax: 55 (61) 7814-2981 \\ E-mail: zancanaro@unb.br
}

Como citar este artigo/How to cite this article: Zancanaro PCQ, Isaac AR, Garcia LT, Costa IMC. Esclerodermia localizada na criança: aspectos clínicos, diagnósticos e terapêuticos. An Bras Dermatol. 2009;84(2):161-72. 